\title{
Synthesis and Characterization of Nonsymmetric Liquid Crystal Dimer Containing Biphenyl and Azobenzene Moiety
}

\author{
S. Sandy Subala, ${ }^{1}$ B. Syama Sundar, ${ }^{1}$ and S. Sreehari Sastry ${ }^{2}$ \\ ${ }^{1}$ Department of Chemistry, Acharya Nagarjuna University, Nagarjuna Nagar, Guntur 522510, India \\ ${ }^{2}$ Department of Physics, Acharya Nagarjuna University, Nagarjuna Nagar, Guntur 522510, India \\ Correspondence should be addressed to S. Sandy Subala; sandysubala@yahoo.co.in
}

Received 29 February 2012; Revised 24 July 2012; Accepted 7 August 2012

Academic Editor: Ewa Schab-Balcerzak

Copyright (c) 2013 S. Sandy Subala et al. This is an open access article distributed under the Creative Commons Attribution License, which permits unrestricted use, distribution, and reproduction in any medium, provided the original work is properly cited.

\begin{abstract}
Calamitic liquid crystalline dimer containing azobenzene moiety and a decyloxy biphenyl linked by flexible spacers $\{4$-[7-(4'decyloxy-biphenyl-4-yloxy)-alkyloxy]-phenyl\}-(4-decyl-phenyl)-diazene has been synthesized and characterized by spectroscopic methods. The transition temperatures and phase behaviours were studied by Differential Scanning Calorimeter (DSC) and Polarizing Optical Microscope (POM). The synthesized compounds exhibited enantiotropic liquid crystal phase with higher spacer display nematic and smectic $\mathrm{C}$ phases while lower spacer shows nematic and smectic A phases.
\end{abstract}

\section{Introduction}

The liquid crystalline state is characterized by the orientational ordering of its constituent molecules, and transitions between different mesophases are accompanied by changes in the local molecular order. These transitions are usually marked by changes in various anisotropic properties, but depending on the order of the transition they may also be accompanied by changes in scalar quantities such as enthalpy content or density. The alternations in density and thermal expansivity which accompany the nematic to isotropic phase transition have been the subjects of a number of investigations [1-7]. The architecture and functionalization are the essential aspects in the molecular engineering of liquid crystal for the control of important physical properties such as optical anisotropy, dielectric anisotropy, supramolecular order, and thermal stability of mesophases. The initial interest in liquid crystal dimers arose, therefore, from their potential use as model compounds with which to understand the more complex polymeric systems. It quickly became apparent, however, that the dimers themselves were of significant interest in their own right as they exhibited quite different behaviour to conventional low molar mass liquid crystals [7, 8]. The liquid crystal dimer consists of mesogenic units separated by flexible spacers, normally alkyl chains [9]. Nonsymmetry is usually introduced in to a dimer by connecting two different mesogenic units, by changing the length of the terminal chains or by using different terminal groups or varying the length and parity of spacer group [10]. These polymers have been the focus of considerable research interest not only for their technological potential [11] but also on a more fundamental level because they exhibit a range of unusual liquid crystalline behaviour. This includes the dramatic dependence of their transitional properties on the length and parity of the flexible spacers linking the mesogenic units [12], the observation of a nematic-nematic transition [13], and the occurrence of alternating smectic phases [14]. Particularly, molecules containing azo groups are well known to show reversible isomerization transformations upon irradiation with ultraviolet and visible light $[15,16]$. The azobenzene class of chromophores is characterized by the azo linkage $(-\mathrm{N}=\mathrm{N}-)$ that bridges two phenyl rings. This extended aromatic structure gives rise to azobenzene's intense optical absorption and related optical properties [17]. Strictly, "azobenzene" refers only to the parent molecule, though the term is now frequently used to refer to the entire class of substituted azo molecules. Biphenyl is a widely used building block for the construction of calamitic liquid crystals. It acts as a supportive entity for hermochromism. 
TABLE 1: Phase transitions temperatures $\left({ }^{\circ} \mathrm{C}\right)$ and the corresponding enthalpies $(\mathrm{J} / \mathrm{g})$ of liquid crystalline dimers.

\begin{tabular}{|c|c|c|c|c|c|c|c|c|c|c|}
\hline Compound & $n$ & $\mathrm{Cr}$ & Heating/cooling & $\mathrm{SmC}$ & Heating/cooling & SmA & Heating/cooling & $\mathrm{N}$ & Heating/cooling & $\mathrm{I}$ \\
\hline \multirow{2}{*}{ C-4 } & \multirow{2}{*}{4} & \multirow{2}{*}{$\cdot$} & $152.7[17.2]$ & \multirow{2}{*}{ - } & \multirow{2}{*}{ - } & \multirow[t]{2}{*}{ - } & $166.1[3.2]$ & \multirow[t]{2}{*}{ • } & $189.3[2.3]$ & . \\
\hline & & & $135.5[15.9]$ & & & & $162.7[2.6]$ & & $186.3[2.6]$ & \\
\hline \multirow{2}{*}{ C-5 } & \multirow{2}{*}{5} & \multirow[t]{2}{*}{ - } & $169.5[23.1]$ & \multirow[t]{2}{*}{ - } & $155.7[2.3]$ & \multirow{2}{*}{ - } & \multirow{2}{*}{ - } & \multirow[t]{2}{*}{$\cdot$} & $175.0[3.1]$ & • \\
\hline & & & $150.4[14.6]$ & & $159.2[3.9]$ & & & & $177.9[2.3]$ & \\
\hline \multirow{2}{*}{ C-6 } & \multirow{2}{*}{6} & \multirow[t]{2}{*}{ - } & $153.0[51.2]$ & \multirow{2}{*}{ - } & \multirow{2}{*}{-} & \multirow[t]{2}{*}{ - } & $163.0[1.9]$ & \multirow[t]{2}{*}{ • } & $179.0[3.6]$ & • \\
\hline & & & $141.3[49.9]$ & & & & $160.7[2.0]$ & & $181.4[2.5]$ & \\
\hline \multirow{2}{*}{$\mathrm{C}-7$} & \multirow{2}{*}{7} & \multirow[t]{2}{*}{ • } & $131.6[34.6]$ & \multirow[t]{2}{*}{ - } & $149.5[4.1]$ & \multirow{2}{*}{-} & \multirow{2}{*}{ - } & \multirow[t]{2}{*}{ - } & $171.4[4.0]$ & $\cdot$ \\
\hline & & & $138.6[29.1]$ & & $152.6[3.6]$ & & & & $173.6[4.7]$ & \\
\hline \multirow{2}{*}{ C- 8} & \multirow{2}{*}{8} & \multirow[t]{2}{*}{ - } & $167.0[19.5]$ & \multirow{2}{*}{ - } & \multirow{2}{*}{-} & \multirow[t]{2}{*}{$\cdot$} & $153.1[3.1]$ & - & $183.0[6.1]$ & $\cdot$ \\
\hline & & & $140.6[21.6]$ & & & & $151.3[1.8]$ & & $179.1[5.8]$ & \\
\hline C-9 & 9 & - & $149.3[39.4]$ & - & $140.4[2.9]$ & - & - & . & $169.3[4.5]$ & • \\
\hline & & & $131.4[25.7]$ & & $143.9[3.1]$ & - & & & $172.3[5.6]$ & \\
\hline C- 10 & 10 & - & $132.8[19.1]$ & - & - & - & $136.7[4.1]$ & • & $168.3[3.5]$ & • \\
\hline & & & $119.9[22.4]$ & - & & & $137.1[4.9]$ & & $171.9[4.6]$ & \\
\hline C-11 & 11 & - & $112.9[20.3]$ & - & $142.5[3.0]$ & - & - & - & $167.0[2.9]$ & $\cdot$ \\
\hline & & & $124.4[27.8]$ & & $139.6[4.9]$ & - & & & $165.4[3.7]$ & \\
\hline C-12 & 16 & - & $112.5[19.1]$ & - & 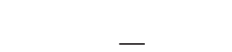 & - & $134.5[2.4]$ & - & $163.5[4.3]$ & \\
\hline & 10 & & 99.4 [30.9] & & & & $137.4[3.0]$ & & 162.2 [3.9] & \\
\hline
\end{tabular}

In the study described here, we combine azobenzene and alkoxy biphenyl units through flexible alkyl spacer to produce a calamitic nonsymmetric liquid crystalline dimer with the aim to study the liquid crystalline properties.

\section{Results and Discussions}

The identification of the mesophases and determination of phase transition temperatures were achieved with the help of polarizing optical microscope (POM). The sample placed between a clean untreated glass slide and a cover slip was used for this particular study. The mesophase assignment was based on the observation of birefringence and fluidity when examined under POM. To confirm the transition temperatures and to determine their associated enthalpies, a differential scanning calorimeter (DSC) calibrated using pure indium was employed. The peaks observed in DSC traces (obtained at a rate of $10^{\circ} \mathrm{C} / \mathrm{min}$.) due to phase transition were reproducible and peak temperatures were consistent with those deduced from the optical experiments.

In an effort to understand the structure-property relations the chain lengths of the paraffinic spacer have been varied and both the terminal alkyl and alkoxy tails have been unvaried. The thermal behaviour and phase transition temperatures of the dimeric compounds are summarized in Table 1. It is clear from Table 1 that all the dimers exhibit enantiotropic mesomorphism, especially the nematic $(\mathrm{N})$ behavior. The DSC thermograms show three exothermal transitions on heating to the isotropic liquid and three endothermic transitions on cooling indicating that the even spacer length of the dimer exhibited enantiotropic behaviour for nematic and smectic A phase and the odd spacer length for nematic and smectic $\mathrm{C}$ phases. The smectic A phase was characterized by the formation of focal conic texture (Figure 1)

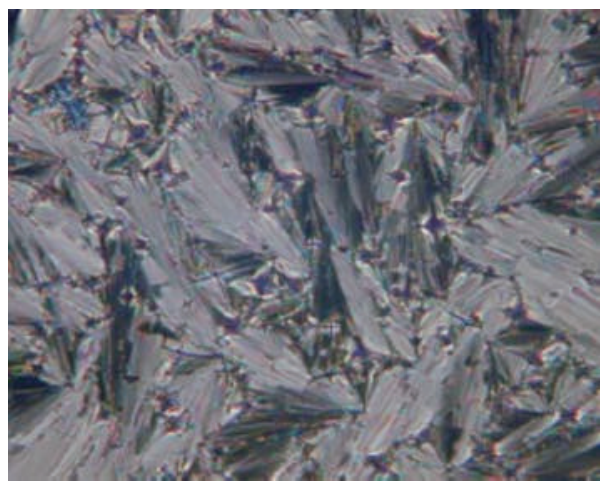

FIGURE 1: Polarising optical microscope textures of SmA focal conic at $160.7^{\circ} \mathrm{C}$ exhibited by the compound C- 6 .

as observed under polarized light. Nematic phases were assigned from their schlieren optical texture containing both types of point singularities, which flashed when subjected to mechanical stress, combined with the high mobility of the phase. The smectic $C$ phase was characterized by the schlieren texture (Figure 2). The increase of the spacer length of the dimer promotes smectic behaviour.

All the dimers show enantiotropic nematic phase. Dimers with even spacer exhibit higher transition temperature than the odd spacer. The odd spacers C-5, C-7, C-9, and C-11 exhibit smectic $\mathrm{C}$ to nematic and nematic to isotropic phase transitions whereas the even spacers C-4, C-6, C-8, C-10, and C-12 exhibit smectic A to nematic and nematic to isotropic phase transitions. The dimers having $\mathrm{C}_{4}$ alkylene spacer show the highest clearing temperatures followed by the dimers with $\mathrm{C}_{8}$ and $\mathrm{C}_{6}$ alkylene spacers. All the synthesized liquid crystal compounds are highly stable. The effects of the spacer length on the transition temperatures and phase behaviour 


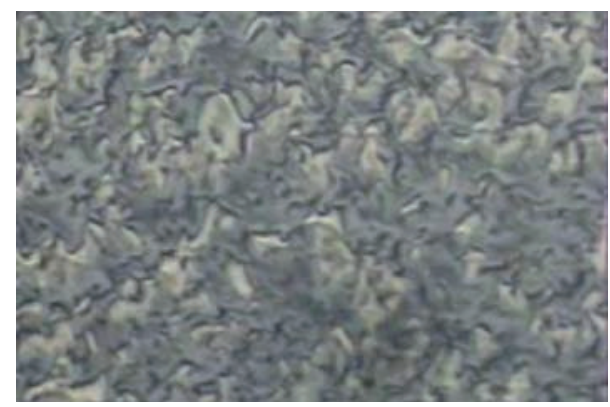

FIgURe 2: Polarising optical microscope textures of SmC schlieren at $139.6^{\circ} \mathrm{C}$ exhibited by the compound C- 11 .

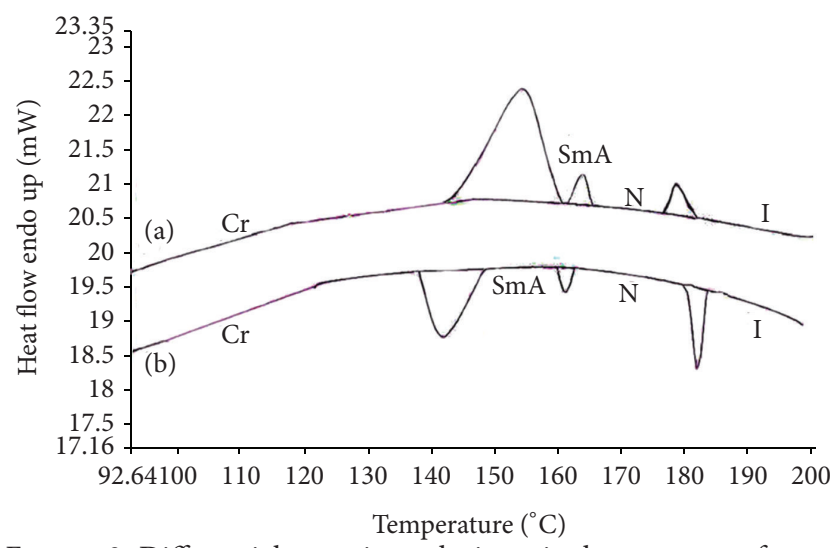

FIGURE 3: Differential scanning calorimetric thermogram of compound C- 6 obtained at a rate of $10^{\circ} \mathrm{C} \mathrm{min}^{-1}$ : (a) heating cycle (b) cooling cycle.

observed in this series are in accord with those observed for conventional low molar mass mesogens. As a representative case the DSC scans obtained at a rate of $10^{\circ} \mathrm{C} / \mathrm{min}$ for the C- 6 sample are shown in Figure 3.

The effects of the spacer length on the transition temperatures and phase behaviour observed in this series are in accord with those observed for conventional low molar mass mesogens. There is a pronounced odd-even effect in the nematic transition temperatures in which the even members have the greater values and as expected this alternation attenuates with increasing spacer length, whereas the odd spacers have lower transition temperature. In Figure 4, the dependence of the transition temperature of the nematic phase on the spacer length for the C-n series is compared (Scheme 1).

\section{Conclusion}

We have synthesized liquid crystalline dimers possessing two nonsymmetrical entities connected by flexible spacers. Molecular design of this dimer incorporates the biphenyl and azo benzene segments interconnected through methylene spacers. All the compounds exhibit enantiotropic mesophases and the transition temperatures for the even spacers are higher than the odd spacer.

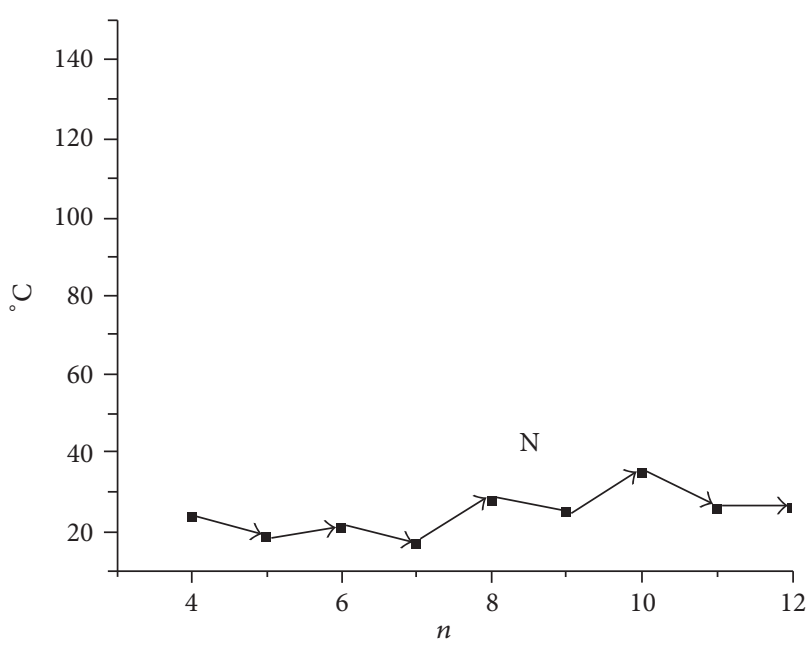

FIGURE 4: Dependence of nematic to isotropic transition temperature for the C-n series.

\section{Experimental}

4.1. Materials. 4-decylaniline, $\alpha$, $\omega$-dibromoalkane, $4,4^{\prime}$ Dihydroxybiphenyl, and 4-decyloxy benzoic acid were purchased from Aldrich Chemical Co. All other solvents and reagents were purchased commercially and used without purification. Dichloromethane and ethanol obtained were purified and dried as per the standard procedures.

4.2. Measurements. IR spectra were recorded on a PerkinElmer Spectrum 1000 FT-IR spectrometer. NMR spectra were recorded using Bruker AMX-400 (400 MHz).The identification of the mesophases and determination of phase transition temperatures were achieved with the help of polarizing optical microscope (Leica DMLP) provided with a heating stage (Mettler FP90). The sample placed between a clean untreated glass slide and a cover slip was used for this particular study. The mesophase assignment was based on the observation of birefringence and fluidity when examined under POM. To confirm the transition temperatures and to determine their associated enthalpies, a differential scanning calorimeter (Perkin Elmer DSC7) calibrated using pure Indium was employed. The peaks observed in DSC traces (obtained at a rate of $10^{\circ} \mathrm{C} / \mathrm{min}$.) due to phase transition were reproducible and peak temperatures were consistent with those deduced from the optical experiments.

4.3. Synthesis of Dimer. The target dimers were purified by column chromatography and recrystallization choosing appropriate mixture of solvents, while the intermediates were purified by column chromatographic technique. Chromatography was performed using either silica gel (100-200, 230-400 mesh). Thin-layer chromatography (TLC) was performed on aluminium sheets precoated with silica gel (Merck, Kieselgel, 60F254). Infra red spectra were recorded on a Perkin-Elmer Spectrum 1000 FTIR spectrometer; the spectral positions (absorption maxima) are given in wave 


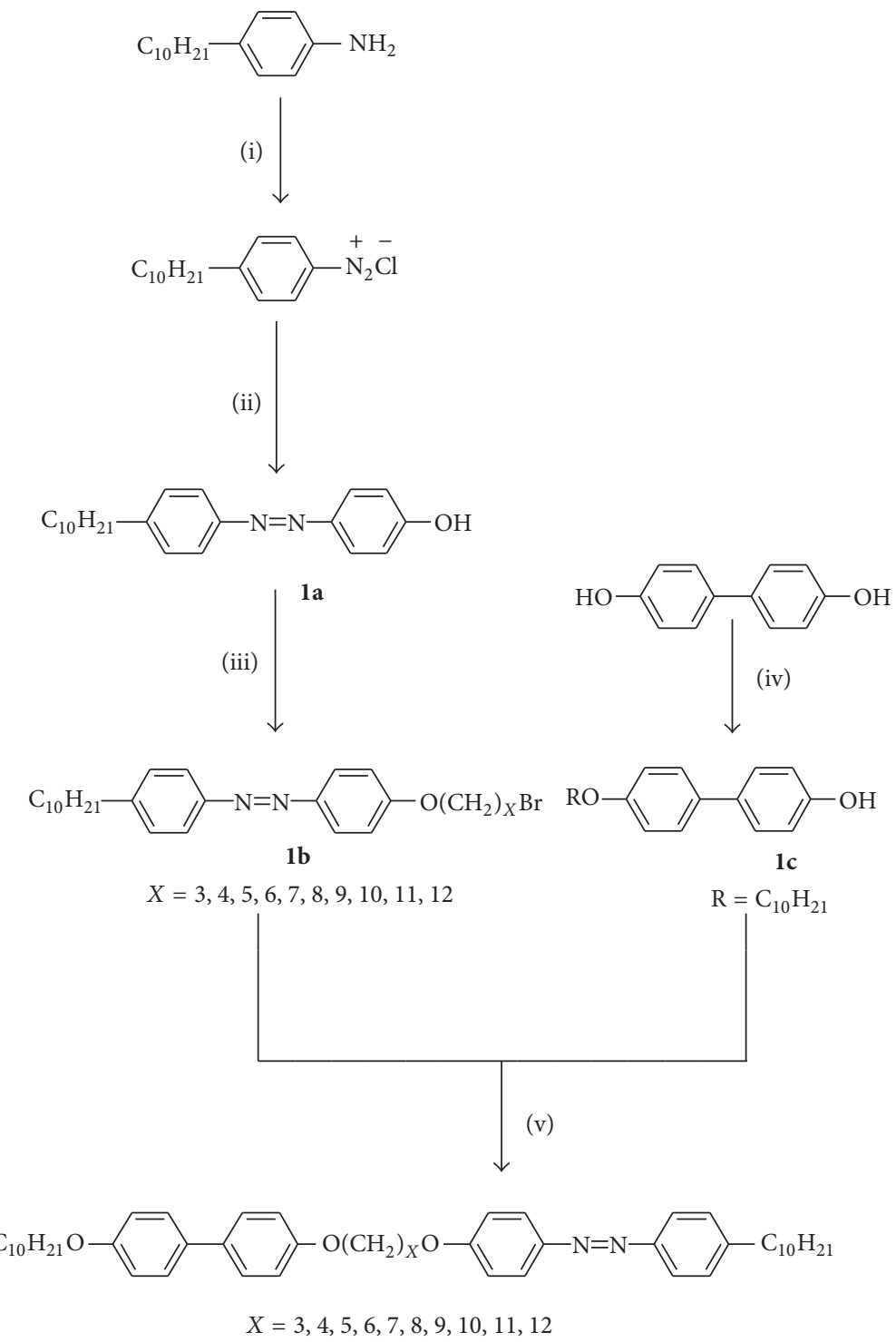

Scheme 1: Scheme C-n. Reagents and condition: (i) sodium nitrite, $\mathrm{HCl}, 0-2^{\circ} \mathrm{C}$; (ii) phenol, aq. $\mathrm{NaOH}, \mathrm{Na}_{2} \mathrm{CO}_{3}, 0-2^{\circ} \mathrm{C}$; (iii) $1,10-$ dibromodecane, anhy. $\mathrm{K}_{2} \mathrm{CO}_{3}$, acetone reflux, $18 \mathrm{~h}$; (iv) 1-bromodecane, anhy. $\mathrm{K}_{2} \mathrm{CO}_{3}$, butanone reflux, $14 \mathrm{~h}$; (v) anhy. $\mathrm{K}_{2} \mathrm{CO}$, $\mathrm{DMF}$ reflux, $22 \mathrm{~h}$.

numbers $\left(\mathrm{cm}^{-1}\right)$. NMR spectra were recorded using Bruker AMX 400 (400 MHz) spectrometer. For ${ }^{1}$ H NMR spectra, the chemical shifts are reported in parts per million relative to $\mathrm{Si}\left(\mathrm{CH}_{3}\right)_{4}$ as an internal standard and coupling constants are presented in hertz. Elemental analysis was performed using a Eurovector model EA 3000 CHNS elemental analyzer.

The azo benzene intermediates (1a and $\mathbf{1 b}$ ) were synthesized in two steps. Firstly, 4-decyl aniline was diazotized using hydrochloric acid, sodium nitrite; sodium hydroxide and sodium carbonate provide the compound (1a). Alkylation of this compound with $\alpha$, $\omega$-dibromoalkane gives the intermediate (1b). The hydroxyl intermediate was synthesized by the O-alkylation of $4,4^{\prime}$-dihydroxybiphenyl using 1 -bromodecane. These upon reacting with the intermediate (1b) furnished the target dimer.
4-Hydroxy-4-decylazo Benzene (1a). 4-Decylaniline (1 g, $0.004 \mathrm{~mol}$ ) was dissolved in $200 \mathrm{~mL}$ of a $1: 1$ mixture of water and acetone and $2.5 \mathrm{~mL}$ of concentrated $\mathrm{HCl}$. To the cooled solution, $\mathrm{NaNO}_{2}(0.29 \mathrm{~g}, 0.004 \mathrm{~mol})$ in $3 \mathrm{~mL}$ of water was added dropwise. The solution was allowed to stand for 30 minutes in an ice bath with temperature $0-2^{\circ} \mathrm{C}$. The resulting diazonium ion solution was added to a cold solution of phenol $(0.4 \mathrm{~g}, 0.004 \mathrm{~mol}), \mathrm{NaOH}(0.33 \mathrm{~g}, 0.004 \mathrm{~mol})$, and $\mathrm{Na}_{2} \mathrm{CO}_{3}(1 \mathrm{~g})$ in $14 \mathrm{~mL}$ of cold water. The precipitate was filtered, dried, and recrystallised from aqueous ethanol. Yield: 84\%; a yellow solid; m.pt: $75^{\circ} \mathrm{C}$. IR (KBr pellet): 3432,2857 , 1652, and $1025 \mathrm{~cm}^{-1} ;{ }^{1} \mathrm{H} \mathrm{NMR}\left(\mathrm{CDCl}_{3}, 400 \mathrm{MHz}\right) \delta: 7.75(\mathrm{~d}$, $J=9.2 \mathrm{~Hz}, 2 \mathrm{H}, \mathrm{Ar}), 7.30(\mathrm{~m}, 4 \mathrm{H}, \mathrm{Ar}), 7.72(\mathrm{~d}, J=8.4 \mathrm{~Hz}, 2 \mathrm{H}$, Ar), $2.66\left(\mathrm{t}, 2 \mathrm{H}, 1 \mathrm{XAr}-\mathrm{CH}_{2}\right), 1.71-1.56\left(\mathrm{~m}, 14 \mathrm{H}, 7 \mathrm{XCH}_{2}\right)$, $1.47\left(\mathrm{~m}, 2 \mathrm{H}, 1 \mathrm{XCH}_{2}\right), 0.95\left(\mathrm{t}, 3 \mathrm{H}, 1 \mathrm{XCH}_{3}\right)$. 
4- $\omega$-Bromoalkyloxy-4-decylazobenzene (1b). Potassium carbonate (1 equiv. $0.046 \mathrm{~mol}$ ) was added to a solution of 4-hydroxy-4-decylazo benzene and $\alpha$, $\omega$-dibromoalkane (4 equiv, $0.070 \mathrm{~mol})$ in acetone $(180 \mathrm{~mL})$. The reaction mixture was refluxed at $50^{\circ} \mathrm{C}$ for $18 \mathrm{~h}$ and then poured into ice cold water. The crude product was extracted in to dichloromethane and dried over sodium sulphate and the solvent removed in vacuo. The crude residue was purified by column chromatography on silica gel (100-200 mesh), eluting with hexane and dichloromethane to afford pure compound.

4- $\omega$-Bromododecyloxy-4-decylazobenzene. Yield: 89\%; a yellow solid; IR ( $\mathrm{KBr}$ pellet): 2810, 1610, 1265, 1039, and $804 \mathrm{~cm}^{-1} ;{ }^{1} \mathrm{H} \mathrm{NMR}\left(\mathrm{CDCl}_{3}, 400 \mathrm{MHz}\right) \delta: 7.71-7.60(\mathrm{~m}, 2 \mathrm{H}$, Ar), 7.29-7.20 (m, 4H, Ar), 6.69-6.62 (m, 2H, Ar), 4.20 $\left(\mathrm{t}, 2 \mathrm{H}, 1 \mathrm{XAr}-\mathrm{OCH}_{2}\right), 3.22\left(\mathrm{t}, 2 \mathrm{H}, 1 \mathrm{XCH}_{2} \mathrm{Br}\right), 2.62(\mathrm{t}, 2 \mathrm{H}$, $\left.1 \mathrm{XAr}-\mathrm{CH}_{2}\right), 1.79-1.73\left(\mathrm{~m}, 38 \mathrm{H}, 19 \mathrm{XCH}_{2}\right), 0.97(\mathrm{t}, 3 \mathrm{H}$, $\left.1 \mathrm{XCH}_{3}\right)$.

4-n-decyloxy-4'-hydroxybiphenyl. 4,4'-Dihydroxybiphenyl ( $1 \mathrm{~g}, 0.005 \mathrm{~mol})$, n-bromodecane $(0.4 \mathrm{~g}, 0.002 \mathrm{~mol})$, and potassium carbonate $(3.5 \mathrm{~g}, 0.026 \mathrm{~mol})$ were refluxed for $14 \mathrm{~h}$ under nitrogen atmosphere using butanone and was filtered through Celite bed when hot. The filtrate was evaporated to get the solid residue and purified by column chromatography on silica gel (100-200 mesh) and recrystallised from ethanol.

Yield: 55\%; IR (KBr pellet): 3305, 2920, 2854, 1619, 1503, 1251, and $832 \mathrm{~cm}^{-1}$; ${ }^{1} \mathrm{H} \mathrm{NMR}\left(\mathrm{CDCl}_{3}, 400 \mathrm{MHz}\right): \delta 7.47(\mathrm{~d}$, $J=8.6 \mathrm{~Hz}, 2 \mathrm{H}, \mathrm{Ar}), 7.43(\mathrm{~d}, J=8.5,2 \mathrm{H}, \mathrm{Ar}), 6.91(\mathrm{~d}, J=8.7$, $2 \mathrm{H}, \mathrm{Ar}), 6.87(\mathrm{~d}, J=8.6,2 \mathrm{H}, \mathrm{Ar}), 4.79(\mathrm{~s}, 1 \mathrm{H}, 1 \mathrm{XOH}), 3.98$ $\left(\mathrm{t}, 2 \mathrm{H}, 1 \mathrm{XAr}-\mathrm{OCH}_{2}\right), 2.0-1.25\left(\mathrm{~m}, 16 \mathrm{H}, 8 \mathrm{XCH}_{2}\right), 0.91(\mathrm{t}, 3 \mathrm{H}$, $\left.1 \mathrm{XCH}_{3}\right)$.

\{4-[7-(4'-Decyloxy-biphenyl-4-yloxy)-alkyloxy]-phenyl\}-(4decyl-phenyl)-diazene. Compound $\mathbf{1 b}$ (1 equiv, $0.0003 \mathrm{~mol}$ ), compound $1 \mathrm{c}$ ( 1 equiv, $0.0004 \mathrm{~mol}$ ), and potassium carbonate ( 3 equiv, $0.0007 \mathrm{~mol}$ ) were refluxed for $22 \mathrm{~h}$ under nitrogen atmosphere using dimethylformamide. Water was added to the reaction mixture at room temperature. The product was filtered and purified by column chromatography on silica gel (100-200 mesh) and recrystallised from dichloromethane/ethanol.

C-3. Yield: 45\%; a yellow solid [found C, 79.77; H, 8.97; N, 4.06. $\mathrm{C}_{47} \mathrm{H}_{64} \mathrm{~N}_{2} \mathrm{O}_{3}$ requires $\left.\mathrm{C}, 80.07 ; \mathrm{H}, 9.15 ; \mathrm{N}, 3.97\right]$. IR ( $\mathrm{KBr}$ pellet): 2854, 2816,1625, 1257, 1041, and $841 \mathrm{~cm}^{-1}$; ${ }^{1} \mathrm{H} \mathrm{NMR}\left(\mathrm{CDCl}_{3}, 200 \mathrm{MHz}\right): 7.86(\mathrm{~d}, J=8.6 \mathrm{~Hz}, 2 \mathrm{H}, \mathrm{Ar})$, $7.79(\mathrm{~d}, J=8.2 \mathrm{~Hz}, 2 \mathrm{H}, \mathrm{Ar}), 7.44-7.41(\mathrm{~m}, 4 \mathrm{H}, \mathrm{Ar}), 7.29$ (d, $J=8.1 \mathrm{~Hz}, 2 \mathrm{H}, \mathrm{Ar}), 6.94(\mathrm{~d}, J=8.4 \mathrm{~Hz}, 2 \mathrm{H}, \mathrm{Ar}), 6.84-6.81$ (m, $4 \mathrm{H}, \mathrm{Ar}), 4.20\left(\mathrm{t}, 6 \mathrm{H}, 3 \mathrm{XOCH}_{2}\right), 2.51\left(\mathrm{t}, 2 \mathrm{H}, 1 \mathrm{XAr}-\mathrm{CH}_{2}\right)$, 1.77-1.74 (m, 32H, 16XCH $\mathrm{XCH}_{2}, 1.24\left(\mathrm{t}, 2 \mathrm{H}, 1 \mathrm{XCH}_{2}\right), 0.99-0.96$ $\left(\mathrm{m}, 6 \mathrm{H}, 2 \mathrm{XCH}_{3}\right)$.

C-4. Yield: 49\%; a yellow solid [found C, 79.93; H, 9.57; N, 3.7. $\mathrm{C}_{48} \mathrm{H}_{66} \mathrm{~N}_{2} \mathrm{O}_{3}$ requires $\left.\mathrm{C}, 80.18 ; \mathrm{H}, 9.25 ; \mathrm{N}, 3.90\right]$. IR $(\mathrm{KBr}$ pellet): $2854,2806,1625,1259,1041$, and $841 \mathrm{~cm}^{-1} ;{ }^{1} \mathrm{H} \mathrm{NMR}$ $\left(\mathrm{CDCl}_{3}, 200 \mathrm{MHz}\right): 7.86(\mathrm{~d}, J=8.6 \mathrm{~Hz}, 2 \mathrm{H}, \mathrm{Ar}), 7.80$ $(\mathrm{d}, J=8.2 \mathrm{~Hz}, 2 \mathrm{H}, \mathrm{Ar}), 7.44-7.41(\mathrm{~m}, 4 \mathrm{H}, \mathrm{Ar}), 7.29(\mathrm{~d}$, $J=8.1 \mathrm{~Hz}, 2 \mathrm{H}, \mathrm{Ar}), 6.94(\mathrm{~d}, J=8.4 \mathrm{~Hz}, 2 \mathrm{H}, \mathrm{Ar}), 6.84-6.81$ $(\mathrm{m}, 4 \mathrm{H}, \mathrm{Ar}), 4.20\left(\mathrm{t}, 6 \mathrm{H}, 3 \mathrm{XOCH}_{2}\right), 2.51-2.53(\mathrm{~m}, 2 \mathrm{H}$, 1XAr- $\left.\mathrm{CH}_{2}\right), 1.77-1.74\left(\mathrm{~m}, 32 \mathrm{H}, 16 \mathrm{XCH}_{2}\right), 1.24-1.22(\mathrm{~m}, 4 \mathrm{H}$, $\left.2 \mathrm{XCH}_{2}\right), 0.99-0.96\left(\mathrm{~m}, 6 \mathrm{H}, 2 \mathrm{XCH}_{3}\right)$.

C-5. Yield: 52\%; a yellow solid [found C, 80.03; H, 9.65; N, 4.12. $\mathrm{C}_{49} \mathrm{H}_{68} \mathrm{~N}_{2} \mathrm{O}_{3}$ requires $\mathrm{C}, 80.28 ; \mathrm{H}, 9.35 ; \mathrm{N}, 3.82$ ]. IR (KBr pellet): 2856, 2806, 1625, 1259, 1051, and $841 \mathrm{~cm}^{-1}$; ${ }^{1} \mathrm{H}$ NMR $\left(\mathrm{CDCl}_{3}, 200 \mathrm{MHz}\right): 7.86(\mathrm{~d}, J=8.6 \mathrm{~Hz}, 2 \mathrm{H}, \mathrm{Ar})$, $7.80(\mathrm{~d}, J=8.2 \mathrm{~Hz}, 2 \mathrm{H}, \mathrm{Ar}), 7.44-7.41(\mathrm{~m}, 4 \mathrm{H}, \mathrm{Ar}), 7.29$ $(\mathrm{d}, J=8.1 \mathrm{~Hz}, 2 \mathrm{H}, \mathrm{Ar}), 6.94(\mathrm{~d}, J=8.4 \mathrm{~Hz}, 2 \mathrm{H}, \mathrm{Ar})$, 6.84-6.81 (m, 4H, Ar), 4.20 (t, 6H, 3XOCH $\mathrm{XOC}_{2}, 2.52-2.55(\mathrm{~m}$, $\left.2 \mathrm{H}, 1 \mathrm{XAr}-\mathrm{CH}_{2}\right), 1.77-1.74\left(\mathrm{~m}, 32 \mathrm{H}, 16 \mathrm{XCH}_{2}\right), 1.24-1.22(\mathrm{~m}$, $\left.6 \mathrm{H}, 3 \mathrm{XCH}_{2}\right), 0.99-0.96\left(\mathrm{~m}, 6 \mathrm{H}, 2 \mathrm{XCH}_{3}\right)$.

C-6. Yield: 55\%; a yellow solid [found C, 80.13; H, 9.74; N, 3.52. $\mathrm{C}_{50} \mathrm{H}_{70} \mathrm{~N}_{2} \mathrm{O}_{3}$ requires $\left.\mathrm{C}, 80.38 ; \mathrm{H}, 9.44 ; \mathrm{N}, 3.75\right]$. IR (KBr pellet): 2856, 2806, 1625, 1259, 1051, and $848 \mathrm{~cm}^{-1}$; ${ }^{1} \mathrm{H} \mathrm{NMR}\left(\mathrm{CDCl}_{3}, 200 \mathrm{MHz}\right): 7.86(\mathrm{~d}, J=8.6 \mathrm{~Hz}, 2 \mathrm{H}, \mathrm{Ar})$, $7.80(\mathrm{~d}, J=8.2 \mathrm{~Hz}, 2 \mathrm{H}, \mathrm{Ar}), 7.44-7.41(\mathrm{~m}, 4 \mathrm{H}, \mathrm{Ar}), 7.28$ (d, $J=8.1 \mathrm{~Hz}, 2 \mathrm{H}, \mathrm{Ar}), 6.94(\mathrm{~d}, J=8.4 \mathrm{~Hz}, 2 \mathrm{H}, \mathrm{Ar}), 6.84-6.81$ $(\mathrm{m}, 4 \mathrm{H}, \mathrm{Ar}), 4.20\left(\mathrm{t}, 6 \mathrm{H}, 3 \mathrm{XOCH}_{2}\right), 2.50\left(\mathrm{t}, 2 \mathrm{H}, 1 \mathrm{Xar}-\mathrm{CH}_{2}\right)$, $1.77-1.74\left(\mathrm{~m}, 32 \mathrm{H}, 16 \mathrm{XCH}_{2}\right), 1.24-1.20\left(\mathrm{~m}, 8 \mathrm{H}, 4 \mathrm{XCH}_{2}\right)$, $0.99-0.96\left(\mathrm{~m}, 6 \mathrm{H}, 2 \mathrm{XCH}_{3}\right)$.

C-7. Yield: 64\%; a yellow solid [found C, 80.67; H, 9.83; N, 3.42. $\mathrm{C}_{51} \mathrm{H}_{72} \mathrm{~N}_{2} \mathrm{O}_{3}$ requires $\left.\mathrm{C}, 80.48 ; \mathrm{H}, 9.53 ; \mathrm{N}, 3.68\right]$. IR ( $\mathrm{KBr}$ pellet): 2856, 2806, 1625, 1260, 1051, and $841 \mathrm{~cm}^{-1}$; ${ }^{1} \mathrm{H} \mathrm{NMR}\left(\mathrm{CDCl}_{3}, 200 \mathrm{MHz}\right): 7.86(\mathrm{~d}, J=8.6 \mathrm{~Hz}, 2 \mathrm{H}, \mathrm{Ar})$, $7.80(\mathrm{~d}, J=8.2 \mathrm{~Hz}, 2 \mathrm{H}, \mathrm{Ar}), 7.44-7.41(\mathrm{~m}, 4 \mathrm{H}, \mathrm{Ar}), 7.28$ $(\mathrm{d}, J=8.1 \mathrm{~Hz}, 2 \mathrm{H}, \mathrm{Ar}), 6.94(\mathrm{~d}, J=8.4 \mathrm{~Hz}, 2 \mathrm{H}, \mathrm{Ar})$, 6.84-6.81 (m, 4H, Ar), $4.20\left(\mathrm{t}, 6 \mathrm{H}, 3 \mathrm{XOCH}_{2}\right), 2.49-2.52(\mathrm{~m}$, $\left.2 \mathrm{H}, 1 \mathrm{XAr}-\mathrm{CH}_{2}\right), 1.77-1.74\left(\mathrm{~m}, 32 \mathrm{H}, 16 \mathrm{XCH}_{2}\right), 1.24-1.20(\mathrm{~m}$, $\left.10 \mathrm{H}, 5 \mathrm{XCH}_{2}\right), 0.99-0.96\left(\mathrm{~m}, 6 \mathrm{H}, 2 \mathrm{XCH}_{3}\right)$.

C-8. Yield: 67\%; a yellow solid [found C, 80.28; H, 9.83; N, 3.91. $\mathrm{C}_{52} \mathrm{H}_{74} \mathrm{~N}_{2} \mathrm{O}_{3}$ requires $\mathrm{C}, 80.57 ; \mathrm{H}, 9.62 ; \mathrm{N}, 3.61$ ]. IR (KBr pellet): 2856, 2806, 1635, 1260,1051, and $841 \mathrm{~cm}^{-1}$; ${ }^{1} \mathrm{H} \mathrm{NMR}\left(\mathrm{CDCl}_{3}, 200 \mathrm{MHz}\right): 7.86(\mathrm{~d}, J=8.6 \mathrm{~Hz}, 2 \mathrm{H}, \mathrm{Ar})$, $7.80(\mathrm{~d}, J=8.2 \mathrm{~Hz}, 2 \mathrm{H}, \mathrm{Ar}), 7.44-7.41(\mathrm{~m}, 4 \mathrm{H}, \mathrm{Ar}), 7.28$ (d, $J=8.1 \mathrm{~Hz}, 2 \mathrm{H}, \mathrm{Ar}), 6.95(\mathrm{~d}, J=8.4 \mathrm{~Hz}, 2 \mathrm{H}, \mathrm{Ar}), 6.84-6.81$ (m, $4 \mathrm{H}, \mathrm{Ar}), 4.20\left(\mathrm{t}, 6 \mathrm{H}, 3 \mathrm{XOCH}_{2}\right), 2.54\left(\mathrm{t}, 2 \mathrm{H}, 1 \mathrm{XAr}-\mathrm{CH}_{2}\right)$, 1.77-1.74 (m, 32H, $\left.16 \mathrm{XCH}_{2}\right), 1.24-1.20\left(\mathrm{~m}, 12 \mathrm{H}, 6 \mathrm{XCH}_{2}\right)$, 0.99-0.96 (m, 6H, 2XCH $\mathrm{XCH}_{3}$.

C-9. Yield: 70\%; a yellow solid [found C, 80.47; H, 9.45; N, 3.67. $\mathrm{C}_{53} \mathrm{H}_{76} \mathrm{~N}_{2} \mathrm{O}_{3}$ requires $\left.\mathrm{C}, 80.66 ; \mathrm{H}, 9.71 ; \mathrm{N}, 3.55\right]$. IR ( $\mathrm{KBr}$ pellet): 2856, 2806, 1635, 1260, 1051, and $841 \mathrm{~cm}^{-1}$; ${ }^{1} \mathrm{H} \mathrm{NMR}\left(\mathrm{CDCl}_{3}, 200 \mathrm{MHz}\right): 7.86(\mathrm{~d}, J=8.6 \mathrm{~Hz}, 2 \mathrm{H}, \mathrm{Ar})$, $7.80(\mathrm{~d}, J=8.2 \mathrm{~Hz}, 2 \mathrm{H}, \mathrm{Ar}), 7.44-7.41(\mathrm{~m}, 4 \mathrm{H}, \mathrm{Ar}), 7.28(\mathrm{~d}$, $J=8.1 \mathrm{~Hz}, 2 \mathrm{H}, \mathrm{Ar}), 6.95(\mathrm{~d}, J=8.4 \mathrm{~Hz}, 2 \mathrm{H}, \mathrm{Ar}), 6.84-6.81$ $(\mathrm{m}, 4 \mathrm{H}, \mathrm{Ar}), 4.20\left(\mathrm{t}, 6 \mathrm{H}, 3 \mathrm{XOCH}_{2}\right), 2.49\left(\mathrm{t}, 2 \mathrm{H}, 1 \mathrm{XAr}-\mathrm{CH}_{2}\right)$, 1.77-1.74 (m, $\left.32 \mathrm{H}, 16 \mathrm{XCH}_{2}\right), 1.24-1.20\left(\mathrm{~m}, 14 \mathrm{H}, 7 \mathrm{XCH}_{2}\right)$, 0.99-0.96 (m, 6H, 2XCH $)_{3}$.

C-10. Yield: 70\%; a yellow solid [found C, 80.56; H, 9.94; $\mathrm{N}, 3.79$. $\mathrm{C}_{54} \mathrm{H}_{78} \mathrm{~N}_{2} \mathrm{O}_{3}$ requires $\left.\mathrm{C}, 80.75 ; \mathrm{H}, 9.79 ; \mathrm{N}, 3.49\right]$. 
IR (KBr pellet): 2856, 2806, 1635, 1268, 1051, and $841 \mathrm{~cm}^{-1}$; ${ }^{1} \mathrm{HNMR}\left(\mathrm{CDCl}_{3}, 200 \mathrm{MHz}\right): 7.86(\mathrm{~d}, J=8.6 \mathrm{~Hz}, 2 \mathrm{H}, \mathrm{Ar})$, $7.80(\mathrm{~d}, J=8.2 \mathrm{~Hz}, 2 \mathrm{H}, \mathrm{Ar}), 7.44-7.42(\mathrm{~m}, 4 \mathrm{H}, \mathrm{Ar}), 7.28$ (d, $J=8.1 \mathrm{~Hz}, 2 \mathrm{H}, \mathrm{Ar}), 6.96(\mathrm{~d}, J=8.4 \mathrm{~Hz}, 2 \mathrm{H}, \mathrm{Ar}), 6.84-6.81$ $(\mathrm{m}, 4 \mathrm{H}, \mathrm{Ar}), 4.20\left(\mathrm{t}, 6 \mathrm{H}, 3 \mathrm{XOCH}_{2}\right), 2.50\left(\mathrm{t}, 2 \mathrm{H}, 1 \mathrm{XAr}-\mathrm{CH}_{2}\right)$, 1.77-1.74 (m, 32H, 16XCH $), 1.24-1.19\left(\mathrm{~m}, 16 \mathrm{H}, 8 \mathrm{XCH}_{2}\right)$, 0.99-0.96 (m, 6H, 2XCH $\mathrm{XCH}_{3}$.

C-11. Yield: $72 \%$; a yellow solid [found C, 81.14; $\mathrm{H}, 9.72$; $\mathrm{N}, 3.72 . \mathrm{C}_{55} \mathrm{H}_{80} \mathrm{~N}_{2} \mathrm{O}_{3}$ requires $\mathrm{C}, 80.83 ; \mathrm{H}, 9.87 ; \mathrm{N}, 3.43$. IR (KBr pellet): 2856, 2806, 1635, 1268, 1071, and $841 \mathrm{~cm}^{-1}$; ${ }^{1} \mathrm{HNMR}\left(\mathrm{CDCl}_{3}, 200 \mathrm{MHz}\right): 7.85(\mathrm{~d}, J=8.6 \mathrm{~Hz}, 2 \mathrm{H}, \mathrm{Ar})$, $7.81(\mathrm{~d}, J=8.2 \mathrm{~Hz}, 2 \mathrm{H}, \mathrm{Ar}), 7.44-7.41$ (m, 4H, Ar), 7.29 (d, $J=8.1 \mathrm{~Hz}, 2 \mathrm{H}, \mathrm{Ar}), 6.96(\mathrm{~d}, J=8.4 \mathrm{~Hz}, 2 \mathrm{H}, \mathrm{Ar}), 6.84-6.80$ (m, $4 \mathrm{H}, \mathrm{Ar}), 4.20\left(\mathrm{t}, 6 \mathrm{H}, 3 \mathrm{XOCH}_{2}\right), 2.54\left(\mathrm{t}, 2 \mathrm{H}, 1 \mathrm{XAr}-\mathrm{CH}_{2}\right.$ ), $1.77-1.74\left(\mathrm{~m}, 32 \mathrm{H}, 16 \mathrm{XCH}_{2}\right), 1.24-1.19\left(\mathrm{~m}, 18 \mathrm{H}, 9 \mathrm{XCH}_{2}\right)$, 0.99-0.96 (m, 6H, 2XCH 3$)$.

C-12. Yield: 75\%; a yellow solid [found C, 80.78; $\mathrm{H}, 10.12$; $\mathrm{N}, 3.57 . \mathrm{C}_{56} \mathrm{H}_{82} \mathrm{~N}_{2} \mathrm{O}_{3}$ requires $\left.\mathrm{C}, 80.91 ; \mathrm{H}, 9.94 ; \mathrm{N}, 3.37\right]$. IR (KBr pellet): 2862, 2806, 1635, 1268, 1071, and $841 \mathrm{~cm}^{-1}$; ${ }^{1} \mathrm{HNMR}\left(\mathrm{CDCl}_{3}, 200 \mathrm{MHz}\right): 7.86(\mathrm{~d}, J=8.6 \mathrm{~Hz}, 2 \mathrm{H}, \mathrm{Ar})$, $7.80(\mathrm{~d}, J=8.2 \mathrm{~Hz}, 2 \mathrm{H}, \mathrm{Ar}), 7.44-7.41(\mathrm{~m}, 4 \mathrm{H}, \mathrm{Ar}), 7.28(\mathrm{~d}$, $J=8.1 \mathrm{~Hz}, 2 \mathrm{H}, \mathrm{Ar}), 6.96(\mathrm{~d}, J=8.4 \mathrm{~Hz}, 2 \mathrm{H}, \mathrm{Ar}), 6.84-6.81$ (m, $4 \mathrm{H}, \mathrm{Ar}), 4.20\left(\mathrm{t}, 6 \mathrm{H}, 3 \mathrm{XOCH}_{2}\right), 2.53\left(\mathrm{t}, 2 \mathrm{H}, 1 \mathrm{XAr}-\mathrm{CH}_{2}\right)$, $1.77-1.75\left(\mathrm{~m}, 32 \mathrm{H}, 16 \mathrm{XCH}_{2}\right), 1.24-1.19\left(\mathrm{~m}, 20 \mathrm{H}, 10 \mathrm{XCH}_{2}\right)$, 0.99-0.96 (m, 6H, 2XCH 3$)$.

\section{References}

[1] C. T. Imrie, G. R. Luckhurst, D. Demus et al., Eds., Handbook of Liquid Crystals, Vol 2B. Low Molecular Weight Liquid Crystals, Wiley-VCH, Weinheim, 1998.

[2] C. T. Imrie, "Liquid crystal dimers," Structure \& Bonding, vol. 95, pp. 149-192, 1999.

[3] C. K. Ober, J. I. Jin, Q. Zhou, and R. W. Lenz, "Liquid crystal polymers with flexible spacers in the main chain," in Liquid Crystal Polymers I, N. Platé, Ed., vol. 59 of Advances in Polymer Science, pp. 103-146, Springer, Berlin, Germany, 1984.

[4] A. Mori, K. Kubo, M. Takemoto, and S. Ujiie, "Non-symmetric calamitic liquid crystal dimers containing troponoid and benzenoid units," Liquid Crystals, vol. 32, no. 8, pp. 1021-1029, 2005.

[5] G. S. Attard, "Effect of spacer length on the thermal properties of side-chain liquid crystal polymethacrylates," Trends in Polymer Science, vol. 1, pp. 79-86, 1993.

[6] G. Ungar, V. Percec, and M. Zuber, "Liquid crystalline polyethers based on conformational isomerism. 20. Nematicnematic transition in polyethers and copolyethers based on 1-(4-hydroxyphenyl)-2-(2-R-4-hydroxyphenyl)ethane with $\mathrm{R}=$ fluoro, chloro, and methyl and flexible spacers containing an odd number of methylene units," Macromolecules, vol. 25, no. 1, pp. 75-80, 1992.

[7] A. Iwana, H. Janeczek, A. Hreniak, M. Palewicz, and D. Pociecha, "Thermal and current-voltage behaviour of liquid crystal compounds with rod and bent shapes comprising alkoxysemifluorinated and imine segments," Liquid Crystals, vol. 37, no. 8, pp. 1021-1031, 2010.
[8] M. Tokita, K. Osada, and J. Watanabe, “Thermotropic liquid crystals of polyesters having a mesogenic $\mathrm{P}, \mathrm{P}^{\prime}$-bibenzoate unit $\mathrm{X}$. Distinct orientation of molecules in a thin SmCA film stretched from isotropic melt, providing evidence for the biaxiallity of the SmCA phase," Liquid Crystals, vol. 24, no. 3, pp. 477-480, 1998.

[9] T. Ikeda and O. Tsutsumi, "Optical switching and image storage by means of azobenzene liquid-crystal films," Science, vol. 268, no. 5219, pp. 1873-1875, 1995.

[10] S. K. Prasad, K. L. Sandhya, G. G. Nair, U. S. Hiremath, and C. V. Yelamaggad, "Spacer parity dependence of photoinduced effects in liquid-crystalline dimers," Journal of Applied Physics, vol. 92, no. 2, p. 838, 2002.

[11] V. W. Maier and A. Saupe, "Eine einfache molekular-statistische theorie der nematischen kristallinflüssigen phase. Teil II," Zeitschrift für Naturforschung, vol. 15a, pp. 287-292, 1960.

[12] Y. Liu, G. Zhan, X. Zhong, Y. Yu, and W. Gan, "Effect of pipi stacking on the self-assembly of azomethine-type rod-coil liquid crystals," Liquid Crystals, vol. 38, no. 8, pp. 995-1006, 2011.

[13] M. J. Press and A. S. Arrott, "Expansion coefficient of methoxybenzylidene butylaniline through the liquid-crystal phase transition," Physical Review A, vol. 8, no. 3, pp. 1459-1465, 1973.

[14] E. Gulari and B. Chu, "Density of methoxybenzylidene butylaniline about the isotropic-nematic phase transition," The Journal of Chemical Physics, vol. 62, no. 3, pp. 795-797, 1975.

[15] B. Bahadur, Zeitschrift für Naturforschung, vol. 30, p. 1094, 1975.

[16] D. Armitage and F. P. Price, "Volumetric behaviour of Liquid crystal N-p-Cyanobenzylidene-p-octyloxyaniline," Molecular Crystals and Liquid Crystals, vol. 38, no. 1, pp. 229-237, 1976.

[17] L. Hamryszak, H. Janeczek, and E. Schab-Balcerzak, "New thermotropic symmetrical and unsymmetrical azomethine with azobenzene unit and fluorinated alkyl chain: synthesis and characterization," Journal of Molecular Liquids, vol. 165, pp. 12-20, 2012. 

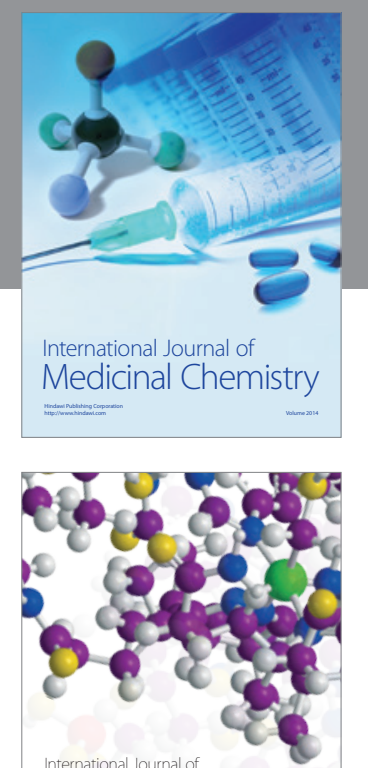

\section{Carbohydrate} Chemistry

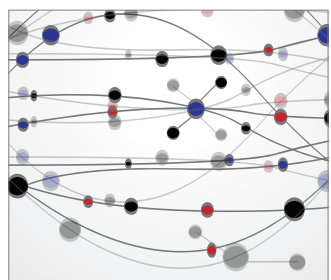

The Scientific World Journal
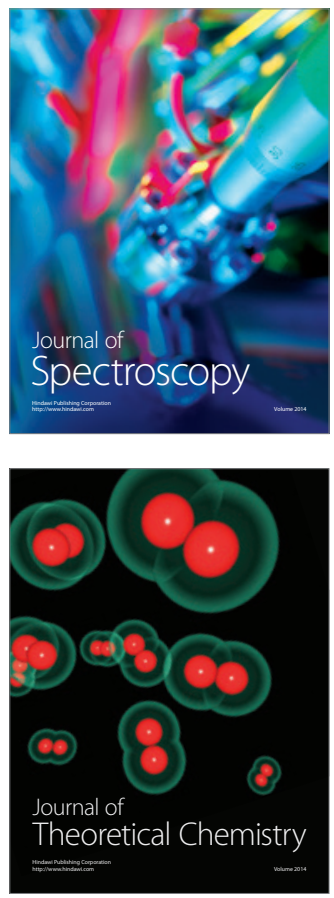
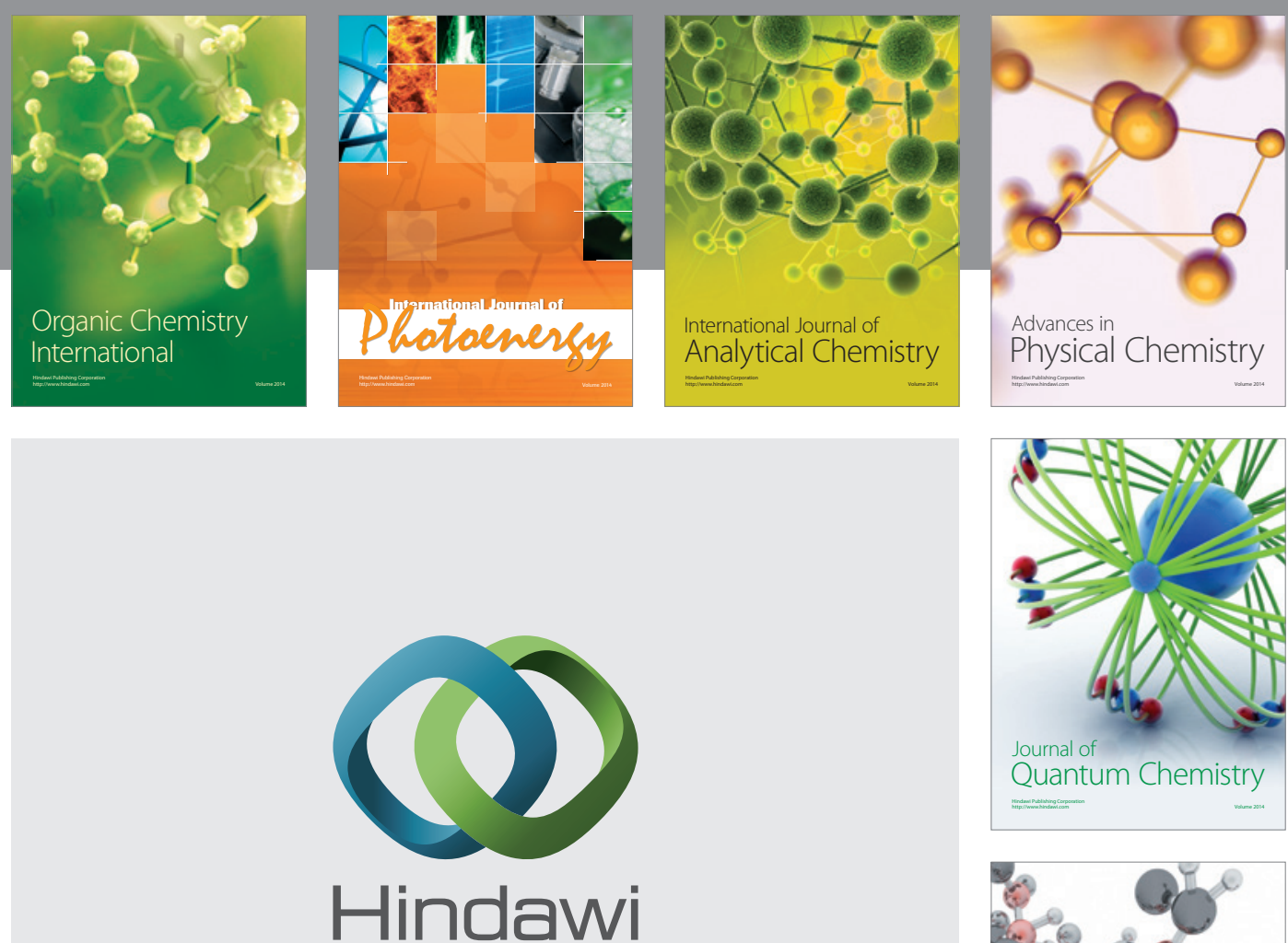

Submit your manuscripts at

http://www.hindawi.com

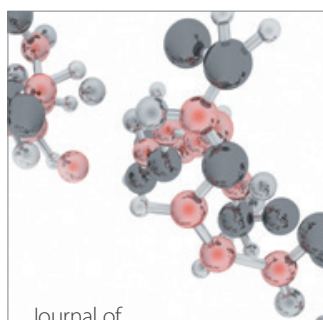

Analytical Methods

in Chemistry

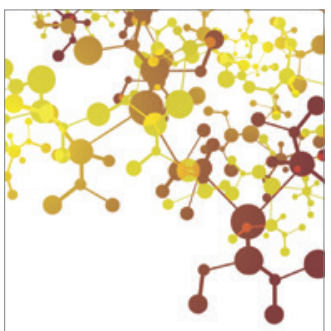

Journal of

Applied Chemistry

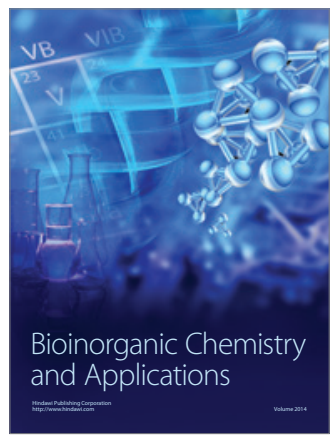

Inorganic Chemistry
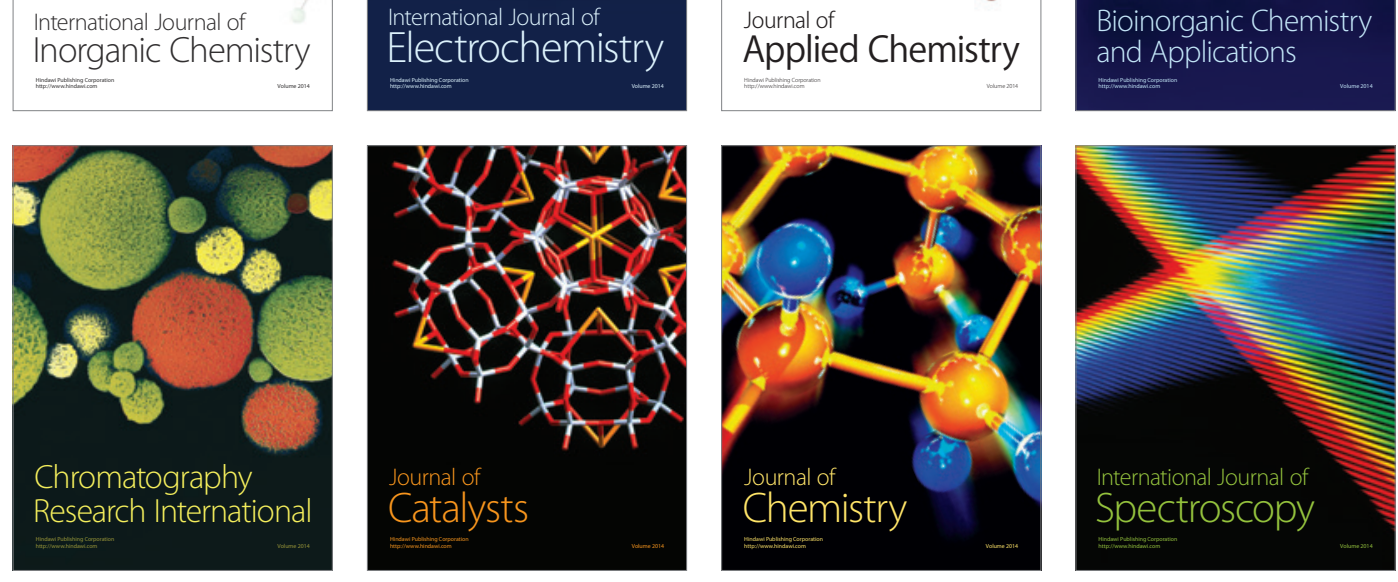